\title{
Diagnostic and therapeutic approach to coexistent chronic obstructive pulmonary disease and obstructive sleep apnea
}

Sanja Jelic

Division of Pulmonary, Allergy, and Critical Care Medicine, Columbia University College of Physicians and Surgeons, New York, NY, USA
Correspondence: Sanja Jelic Columbia University College of Physicians and Surgeons, Division of Pulmonary, Allergy, and Critical Care Medicine, PH8 Center, Room 840, 630 West 168th Street, New York, NY 10032, USA Tel + I 212305759 |

Fax +I 2123057072

Email sj366@columbia.edu

\begin{abstract}
The high prevalence of both obstructive sleep apnea (OSA) and chronic obstructive pulmonary disease (COPD) in Western societies is well documented. However, OSA frequently remains unrecognized and untreated among patients with COPD. Patients with both conditions have a greater risk for fatal and nonfatal cardiovascular events compared with patients with COPD or OSA alone. Efficacious treatment with continuous positive airway pressure reduces the risk of cardiovascular complications in patients with OSA. The aim of the present review is to discuss the diagnostic approach to patients with both conditions and to delineate the benefits of timely recognition and treatment of OSA in patients with COPD.
\end{abstract}

Keywords: chronic obstructive pulmonary disease, obstructive sleep apnea, continuous positive airway pressure, nocturnal arterial oxyhemoglobin desaturation

\section{Introduction}

The high prevalence of obstructive sleep apnea (OSA) among patients with chronic obstructive pulmonary disease (COPD) parallels the recent increase in obesity in Western societies. The prevalence of OSA among patients with COPD is similar to that of the general population ( $22 \%$ and $25 \%-29 \%$, respectively) while the prevalence of obstructive airway disease among patients with OSA is even higher (29\%-40\%) (Young et al 1993; de Miguel et al 2002; Sanders et al 2003; O’Brien et al 2005). Although the clear common risk factors for such strong association are incompletely understood, the sheer number of patients with COPD and OSA accounts for their frequent coexistence.

Despite well documented high prevalence of both COPD and OSA, the coexistence of both conditions remains frequently unrecognized. Nocturnal arterial oxyhemoglobin desaturation and diurnal hypoxemia and hypercapnia are more pronounced in patients with coexistent COPD and OSA than in patients with either condition alone (Chaouat et al 1995; Sanders et al 2003). Patients with both conditions have a greater risk for pulmonary hypertension and right heart failure compared with patients with COPD or OSA alone (Bradley et al 1985; Chaouat et al 1995). Most importantly, patients with coexistent COPD and OSA have increased mortality compared with patients with OSA alone (Lavie et al 1995; Chaouat et al 1999). Treatment with continuous positive airway pressure (CPAP) reduces the risk of fatal and nonfatal cardiovascular events in patients with OSA (Marin et al 2005). Therefore, early recognition and prompt treatment of coexistent OSA in patients with COPD is essential for reducing morbidity and mortality in these patients.

The present review discusses the diagnostic challenges presented by the coexistence of COPD and OSA, and emphasizes the importance of timely identification and treatment of OSA in patients with COPD. 


\section{Diagnostic challenges in patients with coexistent COPD and OSA}

Patients with OSA experience repetitive episodes of hypoxia/ reoxygenation during apneas and hypopneas while asleep resulting in increased arterial stiffness, blood pressure, and sympathetic activity (Carlson et al 1993; Nieto et al 2000; Jelic et al 2002). Untreated OSA increases risk for hypertension, heart failure, stroke, and increased mortality (He et al 1988; Peker et al 1999; Peppard et al 2000; Yaggi et al 2005). Patients with untreated severe OSA have a higher incidence of fatal and nonfatal cardiovascular events than patients treated with CPAP after adjustment for pre-existing cardiovascular risk factors (Marin et al 2005).

COPD is an independent risk factor for increased cardiovascular mortality and morbidity. Forced expiratory volume in 1 second $\left(\mathrm{FEV}_{1}\right)$ is as good a predictor of cardiovascular mortality as serum cholesterol (Hole et al 1996). Ischemic heart disease, and not respiratory failure, is the leading cause of death in COPD patients (Pauwels et al 2001). While right heart failure is a well established complication of COPD, the high prevalence of left ventricular (LV) systolic dysfunction in patients with COPD remains unclear. Patients with COPD are not at increased risk for hypertension or LV hypertrophy; however, they consistently show evidence of low-grade systemic inflammation that plays a major role in the pathogenesis of atherosclerosis (Sin et al 2004). Patients with severe COPD and unknown OSA status are 2.18 and 2.74 times more likely to have elevated and highly elevated, respectively, circulating C-reactive protein levels than control subjects (Sin et al 2003). Untreated OSA is associated with systemic inflammation as well (Shamsuzzaman et al 2002). The presence of unrecognized OSA may potentiate systemic inflammation and accelerate progression of coronary atherosclerosis leading to increased cardiovascular morbidity and mortality in COPD patients.

Furthermore, untreated OSA causes excessive daytime sleepiness that leads to increased risk of accidents at work and while operating a motor vehicle (Sassani et al 2004). Utilization of healthcare resources and economic cost are $23 \%$ to $50 \%$ greater among untreated OSA patients compared with the age-matched subjects (Bahammam et al 1999; Smith et al 2002). Efficacious treatment of OSA reduces the cardiovascular morbidity and mortality as well as cost of care (Marin et al 2005). Despite its significant negative impact on public health, OSA remains frequently unrecognized and untreated.

Identifying patients who experience OSA-related arterial oxyhemoglobin desaturation is especially important in COPD patients since more pronounced nocturnal desaturation correlates with the development of pulmonary hypertension and adverse outcomes (McNicholas et al 1984; Bradley et al 1985). The prevalence of OSA among patients with mild COPD equals that among general middle-age population (22\% vs $29 \%$ ) (Sanders et al 2003). An additional quarter of COPD patients with mild daytime hypoxemia suffer from nocturnal oxyhemoglobin desaturation even in the absence of OSA (Fletcher et al 1987). Progressive decline in $\mathrm{FEV}_{1}$ and forced vital capacity (FVC) ratio to below $65 \%$ is associated with accelerated increase in risk of oxyhemoglobin desaturation during sleep (Sanders et al 2003). Taken together, these epidemiological data suggest that nocturnal oxyhemoglobin desaturation either related or unrelated to OSA is present in at least a half of COPD patients without significant daytime hypoxemia.

Current guidelines for evaluation of nocturnal oxyhemoglobin saturation in COPD patients without significant daytime hypoxemia are somewhat restrictive. Measuring nocturnal oxyhemoglobin saturation in COPD patients who have daytime arterial partial pressure of oxygen $\left(\mathrm{PaO}_{2}\right)$ of $55-59 \mathrm{~mm} \mathrm{Hg}$ is not recommended, except in patients with unexplained polycythemia or cor pulmonale (Celli et al 2004). Nocturnal polysomnography is recommended in COPD patients whose symptoms suggest coexistent OSA. This includes COPD patients who have daytime hypercapnia with only moderately reduced $\mathrm{FEV}_{1}$, those who are obese snorers, or those who develop headache after nocturnal oxygen therapy (Flenley 1985; Celli et al 2004). Witnessed apneas while asleep and daytime sleepiness are highly suggestive of OSA (Hiestand et al 2006). However, the presence of concomitant OSA is often difficult to predict from daytime symptoms in patients with COPD. A majority of patients with OSA do not have daytime hypercapnia or headache and some of them are not obese and have no daytime sleepiness (Young et al 1993; Resta et al 2000; Netzer et al 2003; Bradley et al 2007). Pulmonary function testing correlates poorly with nocturnal hypoxemia while moderate oxyhemoglobin desaturation during exercise has a weak predictive value for reduced nocturnal mean and nadir oxyhemoglobin saturation (Mulloy et al 1996). Significant cardiovascular morbidity and mortality associated with untreated OSA that is reversible with CPAP treatment strongly support a more aggressive approach to diagnosis of coexistent OSA among patients with COPD (Marin et al 2005; Bradley et al 2007).

While the paucity of data regarding prevalence of unrecognized OSA in patients with COPD presents a major challenge, the prevalence of unrecognized COPD among 
patients with known OSA has seldom been addressed. Daytime hypercapnia, pulmonary hypertension, and nocturnal tachyarrhythmias may suggest the presence of unrecognized COPD in patients with OSA (Fletcher et al 1987; Chaouat et al 1995; Resta et al 2002; Olmetti et al 2007). Among 265 patients with OSAS, the prevalence of daytime hypercapnia was $27 \%$ in the 30 patients with an associated COPD and $8 \%$ in the 235 patients without COPD (Chaouat et al 1995). Similarly, daytime hypercapnia was observed in $11 \%$ of 1141 OSA patients who were free of COPD and was related to the severity of obesity (Laaban et al 2005). Pulmonary hypertension is observed in $75 \%$ of patients with coexistent OSA and COPD (Fletcher et al 1987) and in only 12\%-20\% of unselected OSA patients (Bradley et al 1985; Krieger et al 1989). Patients with coexistent OSA and COPD are 2.53 times more likely to experience tachyarrhythmias during sleep that patients with OSA alone (Olmetti et al 2007). Patients with OSA who do not carry the diagnosis of COPD but experience daytime hypercapnia, pulmonary hypertension, and/or nocturnal tachyarrhythmias should be screened for the presence of coexistent COPD including the pulmonary function testing.

Considering that approximately half of patients with COPD who have nocturnal hypoxemia have coexistent OSA, clinicians should be vigilant in establishing the diagnosis of OSA in these patients. Readily available and inexpensive nocturnal oxymetry should be performed in COPD patients with mild daytime hypoxemia and/or $\mathrm{FEV}_{1} / \mathrm{FVC}$ ratio below $65 \%$. Patients with COPD who are diagnosed with nocturnal hypoxemia by nocturnal oxymetry should undergo attended nocturnal polysomnography to exclude coexistent OSA as a treatable cause of nocturnal hypoxemia (Figure 1). Timely diagnosis and treatment of coexistent OSA may reduce cardiovascular morbidity and mortality in patients with COPD.

\section{Treatment of coexistent OSA and COPD \\ Effects of CPAP on lung function in coexistent OSA and COPD}

Prospective studies of long-term CPAP therapy reported reduction in airway obstruction and improvement in gas exchange and respiratory muscle strength in patients with COPD and OSA (Mezzanotte et al 1994; Mansfield et al 1999; de Miguel et al 2002). CPAP therapy for 6 months reduces daytime arterial $\mathrm{PaCO}_{2}$ and increases daytime $\mathrm{PaO}_{2}$ and $\mathrm{FVC}$ and $\mathrm{FEV}_{1}$ in these patients (de Miguel et al 2002).
Improvement in spirometry and gas exchange persisted after 18 months of CPAP therapy (de Miguel et al 2002). In contrast, one retrospective study reported decrease in FVC and $\mathrm{FEV}_{1}$ after initiation of long-term CPAP therapy in patients with coexistent COPD and OSA (O'Brien et al 2005). However, study sample was small $(n=35)$ and less than a third of enrolled patients were included in the final analysis. The reasons for incomplete sample analysis and the precise timing of baseline and follow-up pulmonary function testing were not specified making the results of this study difficult to interpret.

The addition of long-term CPAP therapy to standard treatment of COPD patients improves gas exchange, and airflow obstruction while reducing hospitalization rates in patients with COPD and OSA (Mansfield et al 1999; Pauwels et al 2001; GOLD 2005). An improvement in gas exchange with CPAP has important implications when considering longterm oxygen therapy in patients with coexistent COPD and OSA. Among 55 middle-aged men with daytime hypoxemia and coexistent COPD and OSA, only $22 \%$ continued to have daytime $\mathrm{PaO}_{2}<60 \mathrm{mmHg}$ after 6-month CPAP therapy (de Miguel et al 2002). Furthermore, CPAP therapy reduces elevated pulmonary and systemic arterial pressure in OSA (Arias et al 2006; Campos-Rodriguez et al 2007). This emphasizes the importance of timely diagnosis and treatment of coexistent COPD and OSA to prevent development of severe cardiovascular complications.

Possible mechanisms responsible for CPAP mediated improvement in lung function in patients with coexistent COPD and OSA includes counteraction of auto-positive end-expiratory pressure, decreasing respiratory muscle load, and respiratory work of breathing (Petrof et al 1990; Mezzanotte et al 1994). In addition, CPAP may have a mild bronchodilatory effect by ameliorating chronic irritation and hyperresponsiveness of the upper airway caused by snoring and repetitive closing and opening in OSA (Chan et al 1988). Reduction in chronic airway edema results in increased airway diameter after CPAP therapy (Chan et al 1988; Mansfield et al 1999).

Polysomnography identifies previously unrecognized OSA-related severe nocturnal hypoxemia in COPD patients that requires combined treatment with CPAP and supplemental oxygen. The number of prescriptions for long-term oxygen therapy increased 4-fold among 70 COPD patients after nocturnal polysomnography uncovered previously unrecognized OSA-related nocturnal hypoxemia (O’Brien et al 2005). Patients with coexisting COPD and OSA who require long-term oxygen therapy have less severe COPD 


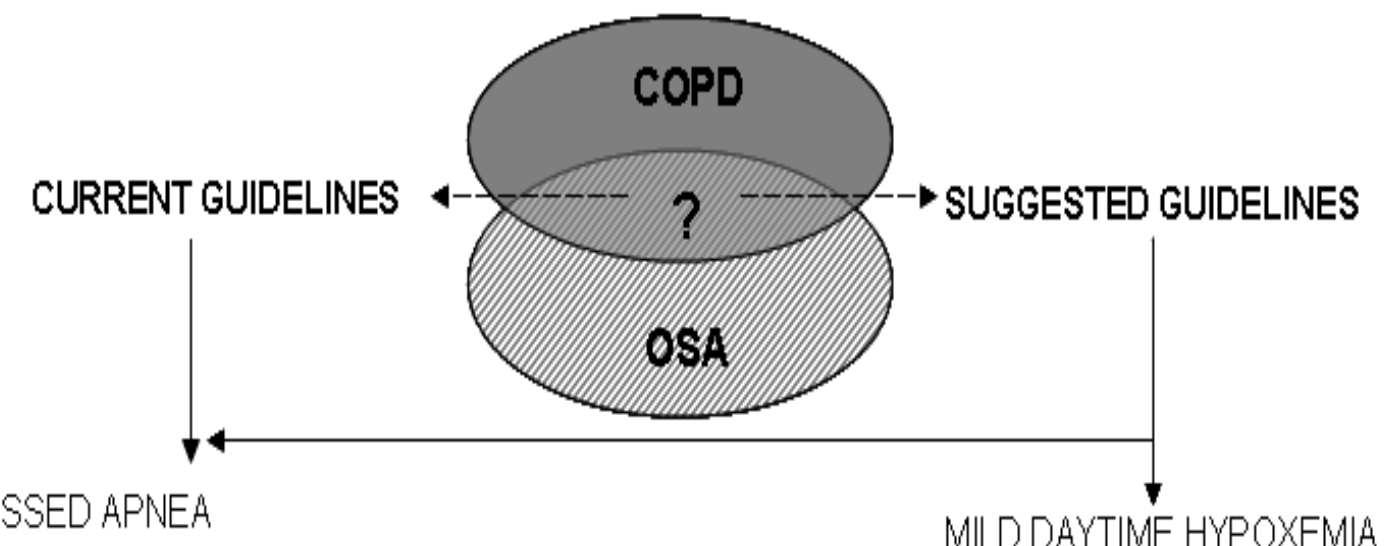

WITNESSED APNEA

DAYTIME SLEEPINESS

OBESITY AND SNORING

DAYTIME HYPERCAPNIA WITH MLLLY Y FEVI

HEADACHE WITH NOCTURNAL OXYGEN THERAPY

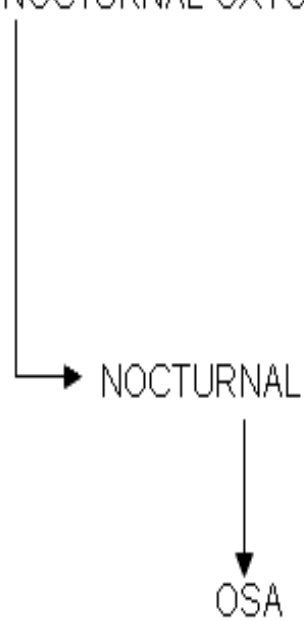

MLDD DAYTIME HYPOXEMIA

ANDIOR

FEVIIFVC RATIO $<65 \%$

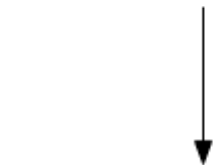

NOCTURNAL OXYMETRY
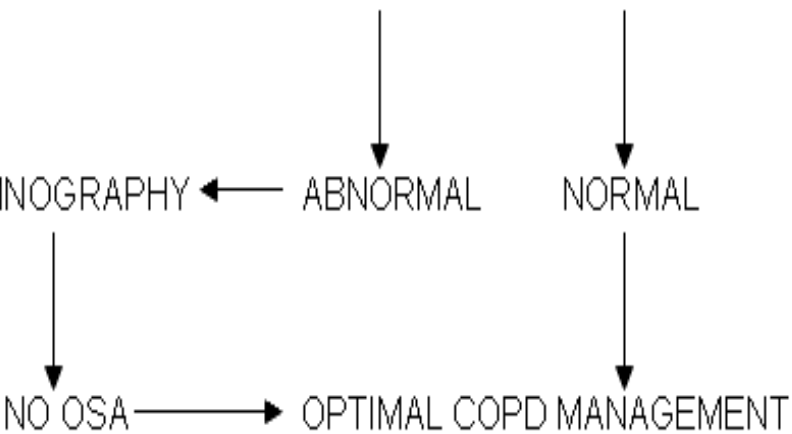

\section{CPAP THERAPY}

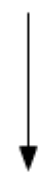

ICARDIOVASCULAR MORBIDITY AND MORTALITY

Figure I Diagnostic and therapeutic approach to coexistent chronic obstructive pulmonary disease (COPD) and obstructive sleep apnea (OSA). Patients with COPD who fulfill the current guidelines criteria for suspected OSA should undergo nocturnal polysomnography without delay. Patients with COPD who do not fulfill the current guidelines criteria for suspected OSA but have mild daytime hypoxemia and/or forced expiratory volume in I second (FEV) and forced vital capacity (FVC) ratio less than 65\% should undergo nocturnal oxymetry. COPD patients with abnormal nocturnal oxymetry should undergo nocturnal polysomnography to establish the diagnosis of possible coexistent OSA. Patients with coexistent COPD and OSA should be treated with continuous positive airway pressure therapy (CPAP) to reduce their cardiovascular morbidity and mortality. 
than patients receiving oxygen therapy due to COPD alone (O'Brien et al 2005). Therefore, all patients with mild to moderate COPD and concomitant daytime hypoxemia should undergo nocturnal oxymetry and/or polysomnography to establish the diagnosis of possible coexistent OSA.

While CPAP therapy is effective in most OSA patients with or without coexistent COPD, bilevel positive airway pressure (PAP) may be useful when patients experience difficulty exhaling against a fixed pressure or have persistent intermittent nocturnal hypoxemia during CPAP titration despite adequate airflow (Kushida et al 2006). This is usually due to COPD-related hypoventilation and ventilationperfusion mismatch. Bilevel PAP delivers a lower expiratory PAP and higher inspiratory PAP thereby augmenting ventilation (Sanders et al 1990; Schafer et al 1998). Some patients with coexistent OSA and COPD experience persistent intermittent nocturnal hypoxemia during CPAP and bilevel PAP titration despite adequate airflow (Sampol et al 1996). PAP can be increased to ameliorate possible residual upper airway resistance. If increased PAP is ineffective or poorly tolerated, supplemental oxygen can be added to PAP to eliminate persistent intermittent nocturnal hypoxemia (Kakkar et al 2007).

The current guidelines suggest the initial follow-up within 1 month after the start of PAP treatment and subsequent yearly and as needed follow-up visits, as well as objective monitoring of CPAP compliance (ATS 1994; Kushida et al 2006). Close follow-up that includes patient education, adjustment of facial interface, and treatment of nasal conditions improves adherence with PAP therapy (Kushida et al 2006).

\section{Long-term oxygen therapy for nocturnal arterial oxyhemoglobin desaturation in COPD in the absence of OSA}

Patients with a resting daytime $\mathrm{PaO}_{2}<55 \mathrm{~mm} \mathrm{Hg}$ are candidates for continuous home oxygen therapy (Celli et al 2004). COPD patients with mild daytime hypoxemia $\left(\mathrm{PaO}_{2}>60 \mathrm{~mm}\right.$ $\mathrm{Hg}$ ) do not qualify for home supplemental oxygen; however, more than a quarter of these patients experience nocturnal arterial oxyhemoglobin desaturation without evidence of OSA (Fletcher et al 1987). Nocturnal arterial oxyhemoglobin desaturation occurs mostly during REM sleep (Fletcher et al 1987). Transient oxyhemoglobin desaturation in COPD probably results from a combination of alveolar hypoventilation and gas exchange abnormalities augmented by REM sleeprelated muscular atonia and changes in respiratory control (Weitzenblum et al 1997). Possible contribution of airflow limitation has been suggested by a modest improvement in oxyhemoglobin desaturation during REM sleep after tiotropium use for 4 weeks in COPD patients without OSA (McNicholas et al 2004). Repetitive alveolar hypoxia and hypoxic vasoconstriction may lead to sustained pulmonary hypertension (Sajkov et al 1999; Fagan 2001). These patients have a greater mortality compared with COPD patients who do not experience nocturnal arterial oxyhemoglobin desaturation (Fletcher et al 1992). Nasal oxygen therapy for 3 years significantly reduced pulmonary artery pressure in COPD patients with nocturnal desaturation (Fletcher et al 1992).

The effect of long-term oxygen therapy on mortality in COPD patients with isolated nocturnal oxyhemoglobin desaturation remains unclear. One study reported a trend toward increased survival among COPD patients with nonapneic nocturnal desaturation who were treated with long-term oxygen therapy (Fletcher et al 1992). Others reported no overall survival benefit with nasal oxygen therapy in these patients (Gorecka et al 1997; Chaouat et al 1999). Low mortality rate in a small study sample precluded definitive conclusion about the effect of nocturnal nasal oxygen therapy on survival in COPD patients with nocturnal arterial oxyhemoglobin desaturation in one study (Chaouat et al 1999). COPD patients with nocturnal arterial oxyhemoglobin desaturation were not identified in another cohort of COPD patients receiving longterm oxygen therapy for mild daytime hypoxemia (Gorecka et al 1997). Pooling together COPD patients with and without nocturnal oxyhemoglobin desaturation may mask a beneficial effect of long-term oxygen therapy on survival in COPD patients with nocturnal oxyhemoglobin desaturation.

In summary, patients with coexistent COPD and OSA have increased morbidity and mortality compared with patients with COPD or OSA alone. Treatment of OSA with CPAP reduces cardiovascular morbidity and mortality in patients with OSA. Clinicians should be vigilant in establishing the diagnosis of OSA in patients with COPD who have nocturnal hypoxemia. Timely diagnosis and treatment of coexistent OSA may reduce cardiovascular morbidity and mortality in patients with COPD.

\section{References}

[ATS] American Thoracic Society. 1994. Indications and standards for use of nasal continuous positive airway pressure (CPAP) in sleep apnea syndromes. Am J Respir Crit Care Med, 150:1738-45.

Arias MA, Garcia-Rio F, Alonso-Fernandez A, et al. 2006. Pulmonary hypertension in obstructive sleep apnoea: effects of continuous positive airway pressure: a randomized, controlled cross-over study. Eur Heart $J$, 27:1106-13

Bahammam A, Delaive K, Ronald J, et al. 1999. Health care utilization in males with obstructive sleep apnea syndrome two years after diagnosis and treatment. Sleep, 22:740-7. 
Bradley TD, Rutherford R, Grossman RF, et al. 1985. Role of daytime hypoxemia in the pathogenesis of right heart failure in the obstructive sleep apnea syndrome. Am Rev Respir Dis, 131:835-9.

Campos-Rodriguez F, Perez-Ronchel J, Grilo-Reina A, et al. 2007. Longterm effect of continuous positive airway pressure on BP in patients with hypertension and sleep apnea Chest, 132:1847-52.

Carlson J, Hedner J, Elam M, et al. 1993. Augmented resting sympathetic activity in awake patients with obstructive sleep apnea. Chest, 103:1763-8.

Celli BR, MacNee W; ATS/ERS Task Force. 2004. Standards for the diagnosis and treatment of patients with COPD: a summary of the ATS/ERS position paper. Eur Respir J, 23:932-46.

Chan CS, Woolcock AJ, Sullivan CE. 1988. Nocturnal asthma: role of snoring and obstructive sleep apnea. Am Rev Respir Dis, 137:1502-4.

Chaouat A, Weitzenblum E, Krieger J, et al. 1995. Association of chronic obstructive pulmonary disease and sleep apnea syndrome. Am J Respir Crit Care Med, 151:82-6.

Chaouat A, Weitzenblum E, Krieger J, et al. 1999. Prognostic value of lung function and pulmonary haemodynamics in OSA patients treated with CPAP. Eur Respir J, 13:1091-6.

de Miguel J, Cabello J, Sanchez-Alarcos JM, et al. Long-term effects of treatment with nasal continuous positive airway pressure on lung function in patients with overlap syndrome. Sleep Breath, 6:3-10.

Fagan KA. 2001. Selected contribution: Pulmonary hypertension in mice following intermittent hypoxia. J Appl Physiol, 90:2502-7.

Flenley DC. 1985. Sleep in chronic obstructive lung disease. Clin Chest Med, 6:651-61.

Fletcher EC, Miller J, Divine GW, et al. 1987. Nocturnal oxyhemoglobin desaturation in COPD patients with arterial oxygen tensions above $60 \mathrm{~mm} \mathrm{Hg}$. Chest, 92:604-8.

Fletcher EC, Schaaf JM, Miller J, et al. 1987. Longterm cardiopulmonary sequelae in patients with sleep apnea and chronic lung disease. Am Rev Respir Dis, 135:525-33.

Fletcher EC, Donner CF, Midgren B, et al. 1992. Survival in COPD patients with a daytime $\mathrm{PaO} 2$ greater than $60 \mathrm{~mm} \mathrm{Hg}$ with and without nocturnal oxyhemoglobin desaturation. Chest, 101:649-55.

Gorecka D, Gorzelak K, Sliwinski P, et al. 1997. Effect of long-term oxygen therapy on survival in patients with chronic obstructive pulmonary disease with moderate hypoxaemia. Thorax, 52:674-9.

[GOLD] Global Initiative for Chronic Obstructive Lung Disease. 2005. Guidelines: Workshop report. Updated September, 2005. Global strategy for diagnosis, management, and prevention of COPD [online]. Accessed October 10, 2007. URL: www.goldcopd.com.

He J, Kryger MH, Zorick FJ, et al. 1988. Mortality and apnea index in obstructive sleep apnea. Experience in 385 male patients. Chest, 94:9-14.

Hiestand DM, Britz P, Goldman M, et al. 2006. Prevalence of symptoms and risk of sleep apnea in the US population: Results from the national sleep foundation sleep in America 2005 poll. Chest, 130:780-6.

Hole DJ, Watt GC, Davey-Smith G, et al. 1996. Impaired lung function and mortality risk in men and women: findings from the Renfrew and Paisley prospective population study, $B M J, 313: 711-15$.

Jelic S, Bartels MN, Mateika JH, et al. 2002. Arterial stiffness increases during obstructive sleep apneas. Sleep, 25:850-5.

Kakkar RK, Berry RB. 2007. Positive airway pressure treatment for obstructive sleep apnea. Chest, 132:1057-72.

Krieger J, Sforza E, Apprill M, et al. 1989. Pulmonary hypertension, hypoxemia and hypercapnia in obstructive sleep apnea patients. Chest, 96:729-37.

Kushida CA, Littner MR, Hirshkowitz M, et al. 2006. Practice parameters for the use of continuous and bilevel positive airway pressure devices to treat adult patients with sleep-related breathing disorders. Sleep, 29:375-80.

Laaban JP, Chailleux E. 2005. Daytime hypercapnia in adult patients with obstructive sleep apnea syndrome in France, before initiating nocturnal nasal continuous positive airway pressure therapy. Chest, $127: 710-15$
Lavie P, Herer P, Peled R, et al. 1995. Mortality in sleep apnea patients: a multivariate analysis of risk factors. Sleep, 18:149-57.

Mansfield D, Naughton MT. 1999. Effects of continuous positive airway pressure on lung function in patients with chronic obstructive pulmonary disease and sleep disordered breathing. Respirology, 4:365-70.

Marin JM, Carrizo SJ, Vicente E, et al. 2005. Long-term cardiovascular outcomes in men with obstructive sleep apnoea-hypopnoea with or without treatment with continuous positive airway pressure: an observational study. Lancet, 365:1046-53.

McNicholas WT, Fitzgerald MX. 1984. Nocturnal deaths among patients with chronic bronchitis and emphysema. Br Med J (Clin Res Ed), 289:878.

McNicholas WT, Calverley PM, Lee A, et al. Tiotropium Sleep Study in COPD Investigators. 2004. Long-acting inhaled anticholinergic therapy improves sleeping oxygen saturation in COPD. Eur Respir $J, 23: 825-31$.

Mezzanotte WS, Tangel DJ, Fox AM, et al. 1994. Nocturnal nasal continuous positive airway pressure in patients with chronic obstructive pulmonary disease. Influence on waking respiratory muscle function. Chest, 106:1100-8.

Mulloy E, McNicholas WT. 1996. Ventilation and gas exchange during sleep and exercise in severe COPD. Chest, 109:387-94.

Netzer NC, Hoegel JJ, Loube D, et al. Sleep in Primary Care International Study Group. 2003. Prevalence of symptoms and risk of sleep apnea in primary care. Chest, 124:1406-14.

Nieto FJ, Young TB, Lind BK, et al. 2000. Association of sleep-disordered breathing, sleep apnea, and hypertension in a large community-based study. Sleep Heart Health Study. JAMA, 283:1829-36.

O'Brien A, Whitman K. Lack of benefit of continuous positive airway pressure on lung function in patients with overlap syndrome. Lung, 183:389-404.

Olmetti F, La Rovere MT, Robbi E, et al. 2007. Nocturnal cardiac arrhythmia in patients with obstructive sleep apnea. Sleep Med, Nov 15 [Epub ahead of print].

Pauwels RA, Buist AS, Calverley PM, et al. 2001. Global strategy for the diagnosis, management, and prevention of chronic obstructive pulmonary disease. NHLBI/WHO Global Initiative for Chronic Obstructive Lung Disease (GOLD) workshop summary. Am J Respir Crit Care Med, 163:1256-76.

Peker Y, Kraiczi H, Hedner J, et al. 1999. An independent association between obstructive sleep apnoea and coronary artery disease. Eur Respir J, 13:179-84.

Peppard PE, Young T, Palta M, et al. 2000. Prospective study of the association between sleep-disordered breathing and hypertension. $N$ Engl J Med, 342:1378-84.

Petrof BJ, Legare M, Goldberg P, et al. 1990. Continuous positive airway pressure reduces work of breathing and dyspnea during weaning from mechanical ventilation in severe chronic obstructive pulmonary disease. Am Rev Respir Dis, 141:281-9.

Resta O, Foschino Barbaro MP, et al. 2000. Hypercapnia in obstructive sleep apnoea syndrome. Neth J Med, 56:215-22.

Resta, O, Barbaro, MPF, Brindicci, C, et al. 2002. Hypercapnia in overlap syndrome: possible determinant factors. Sleep Breath, 6:11-17.

Sajkov D, Wang T, Saunder NA, et al. 1999. Daytime pulmonary hemodynamics in patients with obstructive sleep apnea without lung disease. Am J Respir Crit Care Med, 159:1518-26.

Sampol G, Sagalés MT, Roca A, et al. 1996. Nasal continuous positive airway pressure with supplemental oxygen in coexistent sleep apneahypopnea syndrome and severe chronic obstructive pulmonary disease [abstract]. Eur Respir J, 9:111-16.

Sanders MH, Kern N. 1990. Obstructive sleep apnea treated by independently adjusted inspiratory and expiratory positive airway pressures via nasal mask. Chest, 98:317-24.

Sanders MH, Newman AB, Haggerty CL, et al. 2003. Sleep Heart Health Study. Sleep and sleep-disordered breathing in adults with predominantly mild obstructive airway disease. Am J Respir Crit Care Med, $167: 7-14$ 
Sassani A, Findley LJ, Kryger M, et al. 2004. Reducing motor-vehicle collisions, costs, and fatalities by treating obstructive sleep apnea syndrome. Sleep, 27:453-8.

Schafer H, Ewig S, Hasper E, et al. 1998. Failure of CPAP therapy in obstructive sleep apnea syndrome: predictive factors and treatment with bilevel positive airway pressure. Respir Med, 92:208-15.

Shamsuzzaman AS, Winnicki M, Lanfranchi P, et al. 2002. Elevated Creactive protein in patients with obstructive sleep apnea. Circulation, 105:2462-4.

Sin DD, Man SF. 2003. Why are patients with chronic obstructive pulmonary disease at increased risk of cardiovascular diseases? The potential role of systemic inflammation in chronic obstructive pulmonary disease. Circulation, 107:1514-19.

Sin DD, Hogg J. 2004. Are Patients with chronic obstructive pulmonary disease at increased risk of cardiovascular morbidity and mortality? $C V R \& R, 25: 168-70$.
Smith R, Ronald J, Delaive K, et al. 2002. What are obstructive sleep apnea patients being treated for prior to this diagnosis? Chest, 121:164-72.

Wang H, Parker JD, Newton GE, et al. 2007. Influence of obstructive sleep apnea on mortality in patients with heart failure. $\mathrm{J} \mathrm{Am} \mathrm{Coll} \mathrm{Cardiol}$, 49:1625-31.

Weitzenblum E, Chaouat A, Charpentier C, et al. 1997. Sleep-related hypoxaemia in chronic obstructive pulmonary disease: causes, consequences and treatment. Respiration, 64:187-93.

Yaggi HK, Concato J, Kernan WN, et al. 2005. Obstructive sleep apnea as a risk factor for stroke and death. $N$ Engl J Med, 353:2034-41.

Young T, Palta M, Dempsey J, et al. 1993. The occurrence of sleepdisordered breathing among middle-aged adults. $N$ Engl J Med, Apr $328: 1230-5$ 
